\title{
A Fatal Case of Community Acquired Cupriavidus Pauculus Pneumonia
}

\author{
Emoke Almasy ${ }^{1}$, Janos Szederjesi ${ }^{1 *}$, Paul Rad ${ }^{1}$, Anca Georgescu² \\ 1 Discipline of Intensive Care, University of Medicine and Pharmacy Tîrgu Mureş, Romania \\ 2 Discipline of Infectious Diseases, University of Medicine and Pharmacy Tîrgu Mureş, Romania
}

\begin{abstract}
Introduction: Cupriavidus pauculus is a rarely isolated non-fermentative, aerobic bacillus, which occasionally causes severe human infections, especially in immunocompromised patients. Strains have been isolated from various clinical and environmental sources.

Case presentation: A 67-year-old man was admitted to the Intensive Care Unit with acute respiratory failure. The patient was diagnosed with bilateral pneumonia, pulmonary sepsis and underwent invasive mechanical ventilation. Examination revealed diminished bilateral vesicular breath sounds, fever, intense yellow tracheal secretions, a respiratory rate of $24 /$ minute, a heart rate of $123 /$ minute, and blood pressure of $75 / 55 \mathrm{mmHg}$. Vasoactive treatment was initiated. Investigations revealed elevated lactate and C-reactive protein levels. A chest X-ray showed bilateral infiltration. Parenteral ciprofloxacin and ceftriaxone were administered. Tracheal aspirate culture and blood culture showed bacterial growth of Cupriavidus pauculus. Colistin was added to the treatment. There was a poor clinical response despite repeated blood culture showing negative results. The diagnosis of multiple organ dysfunction syndrome (MODS) caused by C. pauculus was made. The patient died eleven days after admission.

Conclusions: Clinical improvement cannot always be expected in spite of targeted antibiotic therapy. This pathogen should be considered responsible for infections that usually develop in immunocompromised patients.
\end{abstract}

Keywords: sepsis, Cupriavidus pauculus, pneumonia

Received: 14 August 2016 / Accepted: 20 September 2016

\section{INTRODUCTION}

Sepsis is a frequently encountered, life-threatening pathology in intensive care units, induced by infection. The new definitions for sepsis were published in the February issue of JAMA in $2016[1,2]$.

A case series report described a total of thirtytwo cases world-wide of Cupriavidus pauculus infection [3]. Ralstonia, a genus of proteobacteria was described by Yabuuchi, and C. pauculus was firstly described as ralstonia paucula, a rarely isolated, oxidase, catalase, citrate, and urea positive, aerobic non-fermentative motile bacillus [4-6]. There have been reported sepsis cases, such as ventilator associated airway infections, peritonitis, tenosynovitis, and meningitis, caused by this pathogen $[3,7]$.

Ralstonia paucula, strains have been isolated from various clinical sources including hydrotherapy pools, nebulizers, ultrafiltrated water, thermos-regulator res- ervoir water extra-corporeal membrane oxygenation system (ECMO), as well as from environmental samples of pool water, groundwater, tap water, soil, and bottled mineral water $[5,8-10]$.

Although this pathogen occasionally causes serious human infections, especially in immunocompromised patients, thus far Cupriavidus pauculus has rarely been identified as a pathogen in patients [6]. A few reports have described Cupriavidus pauculus infection in children and neonates and several cases have reported positive cultures from intubated Intensive Care Unit (ICU) patients $[3,9,11,12]$.

\section{CASE REPORT}

An intubated 67-year-old man was brought in the emergency department by ambulance with respiratory failure. Within a short time of being admitted to the Intensive Care Unit, County Emergency Clinical Hos-

\footnotetext{
Correspondence to: Janos Szederjesi: Str. Gheorghe Marinescu, Nr. 38, 540139, Tirgu Mures, Romania Tel: +40 265 215 551. E-mail: yangzi37@gmail.com Emoke Almasy: University of Medicine and Pharmacy Tîrgu Mures, Romania, Discipline of Intensive Care, 38 Gheorghe Marinescu street, Tirgu Mures, 540139, Romania Paul Rad: University of Medicine and Pharmacy Tìrgu Mures, Romania, Discipline of Intensive Care, 38 Gheorghe Marinescu street, Tirgu Mures, 540139, Romania

Anca Georgescu: University of Medicine and Pharmacy Tîrgu Mureș, Romania, Discipline of Infectious Diseases, 38 Gheorghe Marinescu street, Tirgu Mures, 540139 , Romania
} 
pital, Tirgu Mureș, Romania, the patient was put on invasive mechanical ventilation. His medical history revealed drug-controlled (carbamazepine, phenobarbital) epileptic seizures and stage 6 Alzheimer disease, which was reported by a family member.

On admission, the patient was diagnosed with severe acute respiratory distress syndrome, bilateral pneumonia with pulmonary sepsis and Stage 2 chronic renal failure. At that time the patient was already on invasive mechanical ventilation, in bi-level airway pressure mode (BIPAP) mode, with a respiratory rate of 14/minute, fraction of inspired oxygen $\left(\mathrm{FiO}_{2}\right): 80 \%$, inspiratory pressure of $20 \mathrm{cmH}_{2} \mathrm{O}$, positive end-expiratory pressure (PEEP): $7 \mathrm{cmH}_{2} \mathrm{O}$. Auscultation revealed a diminished bilateral vesicular murmur with inspiratory crackling. Intense yellow tracheal secretions were observed. He had a Glasgow Coma Score (GCS) of eight. The Full Outline of Unresponsiveness (FOUR) score was nine during residual sedation. His temperature was $38.7^{\circ} \mathrm{C}$, heart rate $123 /$ minute, blood pressure $75 / 55 \mathrm{mmHg}$, and a central venous pressure (CVP) of $11 \mathrm{~cm} \mathrm{H}_{2} \mathrm{O}$. Vasoactive treatment with norepinephrine, $0.4 \mu \mathrm{g} / \mathrm{kg} /$ minute, was initiated.

Further investigations revealed leukocytosis (12100/ $\mathrm{mm}^{3}$ ), neutrophilia (93.6\%), lymphopenia (4.7\%), mild thrombocytopenia $\left(133000 / \mathrm{mm}^{3}\right)$, elevated lactate $(17 \mathrm{mg} / \mathrm{dL})$ and C-reactive protein (CRP) levels $(230 \mathrm{mg} / \mathrm{L})$. CRP level was determined using a BRAHMS KRYPTOR analyzer, TRACE (Time-Resolved Amplified Cryptate Emission) technology.

The prothrombin time was within normal range (12.9 seconds), the alveolar-arterial (A-a) oxygen gradient showed hypoxemia (428 $\mathrm{mmHg}$ ), arterial partial pressure of oxygen $\left(\mathrm{PaO}_{2}\right)$ was $65.9 \mathrm{mmHg}$, and the $\mathrm{PaO}_{2} / \mathrm{FiO}_{2}$ ratio was $82 \mathrm{mmHg}$. Blood sugar levels were slightly higher than normal $(135 \mathrm{mg} / \mathrm{dL})$. The immunoglobulin serum levels were not determined. A chest $\mathrm{x}$-ray showed bilateral interstitial infiltration. The Sequential (Sepsis-Related) Organ Failure Assessment Score (SOFA) was 13, the acute physiology and chronic health evaluation (APACHE) II score was 31. The predicted mortality for this APACHE score is $73 \%$. A diagnosis of sepsis with organ dysfunction was made.

The administration of parenteral ciprofloxacin, 400 mg every 12 hours, and ceftriaxone, $2 \mathrm{~g} /$ day, was commenced. Blood cultures and bacteriological determinations were performed to confirm the diagnosis. The samples were inoculated onto blood agar and triple sugar iron agar (TSI), as a routine procedure. The aspi- rate culture showed bacterial growth after 48 hours of incubation on blood agar and TSI agar, and the gramnegative bacilli were identified as $C$. pauculus by the VITEK 2 Compact automated system. After four days, aerobic blood cultures showed similar growth. Samples were processed using the BacT/Alert 3D (Biomerieux, France) automated haemoculture system. Microbiological findings confirm the diagnosis of C. pauculus sepsis associated with cupriavidus pauculus pneumonia.

The strain was susceptible to ticarcillin, piperacillin, ceftazidime, Cefepime, meropenem, Colistin, and intermediate to ciprofloxacin, resistant to imipenem, amikacin, gentamicin, and tobramycin. Intravenous colistin therapy, 3.000.000 UI eight hourly was added to the existing antibiotic regime from day five.

Despite repeated blood culture showing negative results after five days of targeted antibiotic treatment, the clinical response was poor. The continued presence of cupriavidus pauculus was obtained from repeated cultures of tracheal aspirates. The patient developed liver dysfunction with aspartate aminotransferase $(\mathrm{AST})=1356 \mathrm{U} / \mathrm{L}$, alanine aminotransferase $($ ALT $)=829$ $\mathrm{U} / \mathrm{L}$ and the international normalized ratio (INR) increased spontaneously to 2.67. A prolonged prothrombin time (PT) of 22.7 seconds was recorded. The diagnosis of multiple organ dysfunction syndrome (MODS) caused by $C$. pauculus was confirmed.

The patient's condition worsened. A GCS of six, without sedation, was registered, his central body temperature remained elevated at $38.3^{\circ} \mathrm{C}$, and his mean arterial pressure decreased to $49 \mathrm{mmHg}$. The dosage of norepinephrine was increased to $1.2 \mu \mathrm{g} / \mathrm{kg} / \mathrm{min}$ ute. Hypoxemia persisted with an A-a gradient of 360 $\mathrm{mmHg}$, despite mechanical ventilation. The patient died eleven days after admission.

The patient relatives agreed with the publication of his data. All the procedures were done in concordance with the principles stipulated in the Declaration of Helsinki and followed the approvals released by the institution where the patient was treated.

\section{DISCUSSIONS}

There is a lack of evidence on mortality rates caused by C. pauculus infection, and this is one of the few reported cases $[3,7,12]$.

Sepsis biomarkers like angiopoietin 2, tyrosine kinase 2 , procalcitonin, soluble urokinase-type plasmi- 
nogen receptor and CRP have been shown to be useful in the early diagnosis and prognosis in septic patients $[13,14,15]$.

Stovall et al. were the first to report a C. pauculus infection, which they described as a nosocomial contamination transmitted by an extracorporeal membrane oxygenation (ECMO) equipment [16]. Another case of ventilator-associated pneumonia caused by C. pauculus was reported in Turkey. The successful resolution of the pneumonia was achieved by the administration of imipenem therapy, $2 \mathrm{~g} /$ day for eleven days [7]. The strain isolated in the present case was resistant to imipenem, but susceptible to ticarcillin, piperacillin, ceftazidime, cefepime, meropenem, colistin and intermediate to ciprofloxacin.

The patient received ceftriaxone and ciprofloxacin therapy in the first four days, according to clinical protocols established for community-acquired pneumonia, until cultures proved to be positive and targeted antibiotic therapy could be initiated. Colistin was added to the existing antibiotic treatment in accordance with the bacteriological results and the patient's worsening condition. As no other pathogen was identified, the current antibiotic treatment was continued. Few reports have described combined antibiotic therapy of imipenem, amikacin, imipenem and ciprofloxacin, for this pathogen. In other reported cases positive responses were achieved by a third generation cephalosporin [3]

Aydin et al. (2012) reported a case of a neonate with community-acquired C. pauculus pneumonia [11], Duggal et al. (2013) reported a case of communityacquired C. pauculus meningitis and septicemia in a neonate in India [3]. These two patients responded to treatment and were discharged in good health.

In the present case, blood culture and bacteriological determinations were performed on admission. C. pauculus was present in both the initial blood culture and tracheal aspirate. The patient was in respiratory distress before admission. Nosocomial transmission was ruled out, and the assumption was that it was caused by bacterial contamination water from a well located near his rural home. No environmental cultures were performed, and the exact source of this infection was not identified.

The patient probably was in a poor immunological condition and suffered from Alzheimer's disease. C. pauculus should be considered a possible pathogen in infections that usually develop in immunocompromised patients $[6,7]$. We conclude that the patient's $s$ underlying diseases or complications, like MODS and elevated C-reactive protein level may have contributed to the unfavorable clinical response

\section{-CONCLUSIONS}

This type of infection rarely ends with ICU admission, especially if it is a community-acquired disease. This case shows that clinical improvement cannot always be expected in spite of targeted antibiotic therapy. $C$. pauculus should be considered a potential pathogen in infections that usually develop in immunocompromised patients.

\section{abBREVIATIONS}

$\mathrm{A}-\mathrm{a}=$ alveolar-arterial

ALT = Alanine aminotransferase

APACHE II = Acute Physiology and Chronic Health Evaluation score

AST $=$ Aspartate aminotransferase

BIPAP $=$ Biphasic positive airway pressure

$\mathrm{CVP}=$ Central venous pressure

$\mathrm{ECMO}=$ Extra Corporeal Membrane Oxygenation

FOUR $=$ Full Outline of Unresponsiveness

$\mathrm{FiO} 2=$ Fraction of inspired oxygen

GCS $=$ Glasgow Coma Score

ICU $=$ Intensive Care Unit

MODS = Multiple organ dysfunction syndrome

$\mathrm{PaO} 2=$ Arterial partial pressure of oxygen

PEEP $=$ Positive end-expiratory pressure

SOFA = Sequential (Sepsis Related) Organ Failure Assesment Score

TSI $=$ Triple sugar iron

\section{ACKNOWLEDGEMENT}

I would like to express my deepest appreciation and thanks for the support and help given by my supervisors and the team of the Microbiology Laboratory of the County Emergency Clinical Hospital, Tirgu Mures.

\section{CONFLICT OF INTEREST}

The authors have no conflict of interest regarding the development of this study. 
204 The Journal of Critical Care Medicine 2016;2(4)

\section{REFERENCES}

1. Singer M, Deutschman CS, Seymour CW, et al. The Third International Consensus Definitions for Sepsis and Septic Shock (Sepsis-3). JAMA. 2016;315:801-10. doi: 10.1001/ jama.2016.0287.

2. Copotoiu SM, Copotoiu R. Much Ado About the New Definitions of Sepsis. J Crit Care Med. 2016;2:67-72. DOI: 10.1515/jccm2016-0015.

3. Duggal S, Gur R, Nayar R, Rongpharpi SR, Jain D, Gupta RK. Cupriavidus pauculus (Ralstoniapaucula) concomitant meningitis and septicemia in a neonate: First case report from India. Indian J Med Microbiol. 2013;31:405-9. doi: 10.4103/0255-0857.118871.

4. Yabuuchi E, Kosako Y, Yano I, Hotta H, Nishiuchi Y. Transfer of two Burkholderia and an Alcaligenes species to Ralstonia gen. nov.: proposal of Ralstonia pickettii (Ralston, Palleroni and Doudoroff 1973) comb. nov., Ralstonia solanacearum (Smith 1896) comb. nov. and Ralstonia eutropha (Davis 1969) comb. Microbiol Immunol. 1995;39:897-904.

5. Vandamme P, Goris J, Coenye T, et al. Assignment of Centers for Disease Control group IVc-2 to the genus Ralstonia as Ralstoniapaucula sp. Int J Syst Bacteriol. 1999;49 Pt 2:663-9. DOI: 10.1099/00207713-49-2-663.

6. Mahon CR, Lehman DC, Manuselis G Jr. Textbook of Diagnostic Microbiology. Fourth Edition. Missouri: Maryland Heights. 2011, pp. 499.

7. Taşbakan MS, Yamazhan T, Aydemir S, Bacakoğlu F. A case of ventilator-associated pneumonia caused by Cupriavidus pauculus. Mikrobiyol Bul. 2010;44:127-31.

8. Aspinall, ST, Graham R. Two sources of contamination of a
Available online at: www.jccm.ro

hydrotherapy pool by environmental organisms. J Hosp Infect. 1989;14:285-92.

9. Uzodi AS, Schears GJ, Neal JR, Henry NK. Cupriavidus pauculus bacteremia in a child on extracorporeal membrane oxygenation. ASAIO J. 2014;60:740-1. doi: 10.1097/ MAT.0000000000000120.

10. Foxman B. Molecular Tools and Infectious Disease Epidemiology. California: San Diego. 2012, pp. 173.

11. Aydin B, Dilli D, Zenciroğlu A, Okumuş N, Ozkan S, Tanir G. A case of newborn with community acquired pneumonia caused by Cupriavidus pauculus. Tuberk Toraks. 2012;60:160-2.

12. Azcona-Gutiérrez JM, Buendia-Moreno B, Sáez-Nieto J, LópezBrea-Calvo M. Cupriavidus pauculus isolation in the intensive care unit. Enferm Infecc Microbiol Clin. 2008;26:397-8.

13. Szederjesi J, Almasy E, Lazar A, Huțanu A, Badea I, Georgescu A. An Evaluation of Serum Procalcitonin and C-Reactive Protein Levels as Diagnostic and Prognostic Biomarkers of Severe Sepsis. J Crit Care Med. 2015;1:147-53. DOI: 10.1515/jccm2015-0022.

14. Georgescu AM, Szederjesi J, Voidăzan S, et al. Soluble urokinase-type plasminogen activator receptor (suPAR) - a possible biomarker for bacteremia in sepsis. Rev Romana Med Lab. 2015;23:59-73. DOI:10.1515/rrlm-2015-0002.

15. Szederjesi J, Georgescu A, Santini A, et al. Ang-2, Tie-2, and Ang-2/Tie-2 ratio serum levels as diagnostic and prognostic biomarkers of sepsis in critically ill patients. Rev Romana Med Lab. 2016;24:187-99. DOI:10.1515/rrlm-2016-0007.

16. Stovall SH, Wisdom C, McKamie W, Ware W, Dedman H, Fiser RT. Nosocomial transmission of Cupriavidus pauculus during extracorporeal membrane oxygenation. ASAIO J. 2010;56:4867. doi: 10.1097/MAT.0b013e3181f0c80d. 